\title{
FRAME DUALITY PROPERTIES FOR PROJECTIVE UNITARY REPRESENTATIONS
}

\author{
DEGUANG HAN* AND DAVID LARSON**
}

\begin{abstract}
Let $\pi$ be a projective unitary representation of a countable group $G$ on a separable Hilbert space $H$. If the set $B_{\pi}$ of Bessel vectors for $\pi$ is dense in $H$, then for any vector $x \in H$ the analysis operator $\Theta_{x}$ makes sense as a densely defined operator from $B_{\pi}$ to $\ell^{2}(G)$-space. Two vectors $x$ and y are called $\pi$-orthogonal if the range spaces of $\Theta_{x}$ and $\Theta_{y}$ are orthogonal, and they are $\pi$ - weakly equivalent if the closures of the ranges of $\Theta_{x}$ and $\Theta_{y}$ are the same. These properties are characterized in terms of the commutant of the representation. It is proved that a natural geometric invariant (the orthogonality index) of the representation agrees with the cyclic multiplicity of the commutant of $\pi(G)$. These results are then applied to Gabor systems. A sample result is an alternate proof of the known theorem that a Gabor sequence is complete in $L^{2}\left(\mathbb{R}^{d}\right)$ if and only if the corresponding adjoint Gabor sequence is $\ell^{2}$-linearly independent. Some other applications are also discussed.
\end{abstract}

\section{Introduction and the Main Results}

Frame theory for special systems, including wavelet systems and Gabor systems, has close connections with group representations. The aim of this article is to give a general framework for exploring certain of these connections. We will present some results on a duality property for orthogonal (i.e. strongly disjoint) and weakly equivalent frame-generator vectors for group representations and, more generally, projective unitary representations. Our main results are Theorems 1.2 and 1.3. One consequence is an alternate proof of a known duality result for Gabor systems which is one of the main motivations of this paper. The well-known (Ron-Shen) duality theorem (see section 3) reveals the connection between the frame property of a Gabor family (built on a time-frequency lattice) and the Riesz sequence property of the associated Gabor family (built on the adjoint lattice). This duality theorem also indicates some duality connections between the so-called "orthogonality or strong disjointness" of Gabor families and the commutant of the Gabor operator system. These connections exist for more general systems and our main focus of this paper is to investigate these duality connections for general unitary systems associated with projective unitary representations of countable groups.

Date: November 14, 2011.

2000 Mathematics Subject Classification. Primary 42C15, 46C05, 47B10.

Key words and phrases. Projective unitary representations, frame vectors, Bessel vectors, orthogonal and similar vectors, orthogonality index, duality property , cyclic multiplicity, von Neumann algebras .

(*) The results of this paper were reported by the first named author at the Texas A\&M Linear Analysis and Probability Workshop in 2006 and 2007. He acknowledges the support from the A\&M workshop. $(* *)$ The second author was partially supported by NSF grant DMS-0139386. 
We build onto work begun in $[20,13,14]$. Some of the results in this context on frames and group representations that were proved in [20] and [13] were actually stated for Gabortype frames, group-frames, and group-like frames. We subsequently discovered that we were really proving results for projective group representations. So in this article we will use the "projective" terminology in discussing our earlier results from [20] , [13]. The set of group representations of a group $\mathrm{G}$ is a subset of the set of projective group representations. Moreover, most proofs concerning frames for projective group representations are not much more difficult, if any, than the corresponding proof for group-frames. So in most cases we will only state and prove the projective unitary representation case.

A frame [7] for a Hilbert space $H$ is a sequence $\left\{x_{n}\right\}$ in $H$ with the property that there exist positive constants $A, B>0$ such that

$$
A\|x\|^{2} \leq \sum_{g \in G}\left|\left\langle x, x_{n}\right\rangle\right|^{2} \leq B\|x\|^{2}
$$

holds for every $x \in H$. A tight frame refers to the case when $A=B$, and a Parseval frame refers to the case when $A=B=1$. In the case that (1.1) hold only for all $x \in \overline{\operatorname{span}}\left\{x_{n}\right\}$, then we say that $\left\{x_{n}\right\}$ is a frame sequence. If we only require the right-hand side of the inequality (1.1), then $\left\{x_{n}\right\}$ is called a Bessel sequence.

Recall (cf. [26]) that a projective unitary representation $\pi$ for a countable group $G$ is a mapping $g \rightarrow \pi(g)$ from $G$ into the group $U(H)$ of all the unitary operators on a separable Hilbert space $H$ such that $\pi(g) \pi(h)=\mu(g, h) \pi(g h)$ for all $g, h \in G$, where $\mu(g, h)$ is a scalar-valued function on $G \times G$ taking values in the circle group $\mathbb{T}$. This function $\mu(g, h)$ is then called a multiplier of $\pi$. In this case we also say that $\pi$ is a $\mu$-projective unitary representation. It is clear from the definition that we have

(i) $\mu\left(g_{1}, g_{2} g_{3}\right) \mu\left(g_{2}, g_{3}\right)=\mu\left(g_{1} g_{2}, g_{3}\right) \mu\left(g_{1}, g_{2}\right)$ for all $g_{1}, g_{2}, g_{3} \in G$,

(ii) $\mu(g, e)=\mu(e, g)=1$ for all $g \in G$, where $e$ denotes the group unit of $G$.

Any function $\mu: G \times G \rightarrow \mathbb{T}$ satisfying $(i)-(i i)$ above will be called a multiplier for $G$. It follows from $(i)$ and $(i i)$ that we also have

(iii) $\mu\left(g, g^{-1}\right)=\mu\left(g^{-1}, g\right)$ holds for all $g \in G$.

Examples of projective unitary representations include unitary group representations and Gabor representations (see the definition in section 3) in time-frequency analysis.

Similar to the group unitary representation case, the left and right regular projective representations with a prescribed multiplier $\mu$ for $G$ play important roles here. Let $\mu$ be a multiplier for $G$. For each $g \in G$, we define

$$
\lambda_{g} \chi_{h}=\mu(g, h) \chi_{g h}, \quad h \in G,
$$

and

$$
r_{g} \chi_{h}=\mu\left(h, g^{-1}\right) \chi_{h g^{-1}}, \quad h \in G,
$$

where $\left\{\chi_{g}: g \in G\right\}$ is the standard orthonormal basis for $\ell^{2}(G)$. Clearly, $\lambda_{g}$ and $r_{g}$ are unitary operators on $\ell^{2}(G)$. Moreover, $\lambda$ is a $\mu$-projective unitary representation of $G$ with multiplier $\mu(g, h)$ and $r$ is a projective unitary representation of $G$ with multiplier $\overline{\mu(g, h)}$. The representations $\lambda$ and $r$ are called the left regular $\mu$-projective representation and the right regular $\mu$-projective representation, respectively, of $G$. Let $\mathcal{L}$ and $\mathcal{R}$ be the von Neumann algebras generated by $\lambda$ and $r$, respectively. It is known (cf. [13]), similarly 
to the case for regular group representations, that both $\mathcal{R}$ and $\mathcal{L}$ are finite von Neumann algebras, and that $\mathcal{R}$ is the commutant of $\mathcal{L}$. Moreover, if for each $e \neq u \in G$, either $\left\{v u v^{-1}: v \in G\right\}$ or $\left\{\mu\left(v u v^{-1}, v\right) \overline{\mu(v, u)}: v \in G\right\}$ is an infinite set, then both $\mathcal{L}$ and $\mathcal{R}$ are factor von Neumann algebras.

Given a projective unitary representation $\pi$ of a countable group $G$ on a Hilbert space $H$, a vector $\xi \in H$ is called a complete frame vector (resp. complete tight frame vector, complete Parseval frame vector) for $\pi$ if $\{\pi(g) \xi: g \in G\}$ (here we view this as a sequence indexed by $G$ ) is a frame (resp. tight frame, Parseval frame) for the whole Hilbert space $H$, and is just called a frame vector (resp. tight frame vector, Parseval frame vector) for $\pi$ if $\{\pi(g) \xi: g \in G\}$ is a frame sequence (resp. tight frame sequence, Parseval frame sequence). A Bessel vector for $\pi$ is a vector $\xi \in H$ such that $\{\pi(g) \xi: g \in G\}$ is Bessel. We will use $\mathcal{B}_{\pi}$ to denote the set of all the Bessel vectors of $\pi$.

For any projective representation $\pi$ of a countable group $G$ on a Hilbert space $H$ and $x \in H$, the analysis operator $\Theta_{x}$ for $x$ from $\mathcal{D}\left(\Theta_{x}\right)(\subseteq H)$ to $\ell^{2}(G)$ is defined by

$$
\Theta_{x}(y)=\sum_{g \in G}\langle y, \pi(g) x\rangle \chi_{g},
$$

where $\mathcal{D}\left(\Theta_{x}\right)=\left\{y \in H: \sum_{g \in G}|\langle y, \pi(g) x\rangle|^{2}<\infty\right\}$ is the domain space of $\Theta_{x}$. Clearly, $\mathcal{B}_{\pi} \subseteq \mathcal{D}\left(\Theta_{x}\right)$ holds for every $x \in H$. In the case that $\mathcal{B}_{\pi}$ is dense in $H$, we have that $\Theta_{x}$ is a densely defined and closable linear operator from $\mathcal{B}_{\pi}$ to $l^{2}(G)$ (cf. [12]). Moreover, $x \in \mathcal{B}_{\pi}$ if and only if $\Theta_{x}$ is a bounded linear operator on $H$, which in turn is equivalent to the condition that $\mathcal{D}\left(\Theta_{x}\right)=H$.

It is useful to note that if $\xi$ and $\eta$ are Bessel vectors for $\pi$, then $\Theta_{\eta}^{*} \Theta_{\xi}$ commutes with $\pi(G)$. Thus, if $\xi$ is a complete frame vector for $\pi$, then $\eta:=S_{\xi}^{-1 / 2} \xi$ is a complete Parseval frame vector for $\pi$, where $S_{\xi}=\Theta_{\xi}^{*} \Theta_{\xi}$ and is called the frame operator for $\xi$ (or Bessel operator if $\xi$ is a Bessel vector). Hence, a projective unitary representation has a complete frame vector if and only if it has a complete Parseval frame vector.

There are two basic but useful concepts in frame theory: one is the notion of equivalent (i.e. similar) frames, and the other one is the concept of orthogonal (also called strongly disjoint) frames that was introduced independently by Balan [3] and by the authors in [20]. We refer to some recent papers (cf. $[1,2,3,8,9,10,11,13,14,15,16,18,19,27]$ ) for some applications of orthogonal frames. For our purpose, here we only state the case for projective unitary representations. Suppose $\pi$ is a projective unitary representation of a countable group $G$ on a separable Hilbert space $H$ and suppose that the set $B_{\pi}$ of Bessel vectors for $\pi$ is dense in $H$. This will be our usual setting. We will say that two vectors $x$ and $y$ in $H$ are $\pi$-orthogonal if the ranges of $\Theta_{x}$ and $\Theta_{y}$ are orthogonal, and that they are $\pi$-weakly equivalent if the closures of the ranges of $\Theta_{x}$ and $\Theta_{y}$ are the same. The above $\pi$ orthogonality definition can be extended in an obvious way to a set of several (even infinitely many) vectors. Let $M$ be a subset of a Hilbert space $H$ and $\mathcal{A}$ be a subset of the space $B(H)$ of all the bounded linear operators on $H$. In what follows we will use $[M]$ to denote the closed linear span of $M$ and use $\mathcal{A}^{\prime}$ to denote the commutant $\{T \in B(H): T A=A Y, \forall A \in \mathcal{A}\}$ of $\mathcal{A}$.

Our use of the terminology for strongly disjointness and weakly equivalence is motivated by the following: 
Lemma 1.1. [20] Let $\pi$ be a projective representation of a countable group $G$ on a Hilbert space $H$.

(i) If $\{\pi(g) x: g \in G\}$ and $\{\pi(g) y: g \in G\}$ are frame sequences in $H$, then $x$ and $y$ are $\pi$-weakly equivalent if and only if there exists a bounded invertible operator $S:[\pi(G) x] \rightarrow$ $[\pi(G) y]$ such that $S \pi(g) x=\pi(g) y$ for all $g \in G$. (In this case, the two frame sequences are called equivalent or similar.)

(ii) Suppose that $\left\{\pi(g) \xi_{i}: g \in G\right\}$ is a Parseval frame for $H_{i}:=\left[\pi(G) \xi_{i}\right](i=1, \ldots, N$, and $N$ could be $\infty)$. Then $\left\{\xi_{i}\right\}$ are $\pi$-orthogonal if and only if $\left\{\sum_{i=1}^{n} \oplus \pi(g) \xi_{i}: g \in G\right\}$ is a Parseval frame for $\sum_{i=1}^{N} \oplus H_{i}$.

The following theorem characterizes the $\pi$-orthogonality and $\pi$-weakly equivalence in terms of the commutant of $\pi(G)$. The proof will be given in the next section.

Theorem 1.2. Let $\pi$ be a projective representation of a countable group $G$ on a Hilbert space $H$ such that $\mathcal{B}_{\pi}$ is dense in $H$, and let $x, y \in H$. Then

(i) $x$ and $y$ are $\pi$-orthogonal if and only if $\left[\pi(G)^{\prime} x\right] \perp\left[\pi(G)^{\prime} y\right]$ (or equivalently, $x \perp$ $\left.\pi(G)^{\prime} y\right)$;

(ii) $x$ and $y$ are $\pi$-weakly equivalent if and only if $\left[\pi(G)^{\prime} x\right]=\left[\pi(G)^{\prime} y\right]$,

We remark that a special case of part (ii) of this theorem is known in Gabor analysis which asserts that two Gabor frames (see the definitions in section 3 ) for $L^{2}\left(\mathbb{R}^{d}\right)$ are orthogonal (i.e. strongly disjoint) if and only if the associated Riesz sequences (built via the adjoint lattice) have orthogonal spans. Theorem 1.2 is basic to our approach. We will discuss several other applications of this theorem. One of them is to characterize the orthogonality index, which is defined below.

In [20] we defined the frame multiplicity for a unitary representation of a countable group that admits complete frame vectors (i.e. the representation is equivalent to a subrepresentation of the left regular representation of the group) to be the maximum cardinality of all sets of mutually orthogonal complete frame vectors. That is, the frame multiplicity is the maximal length of a superframe $([3,20])$ for the group representation. This is a natural numerical invariant which played an essential role in classifying these representations and in obtaining our parameterization result for the set of all the frame vectors. In many cases, however, the frame multiplicity does not adequately describe the geometry of the orthogonality properties of the representations. This leads to our definition of orthogonality index (see the definition below). This is a numerical invariant which is always greater than or equal to the frame multiplicity. In the case that $\pi$ admits a complete superframe ( i.e., a superframes which is an orthonomal basis for the direct sum space,) then the two invariants are the same. The two also agrees (indeed both are 1) in the case of a group representation of an abelian group (see Example 3.2 (i)). But they can be different for nonabelian groups (see Example 3.2 (ii)). They do not necessarily agree in the case of projective unitary representations of an abelian group, such as the Gabor representations (see the comment after Corollary 3.1).

For a projective representation $\pi$ of a countable group $G$ on a Hilbert space $H$, we define the decomposition space of $\pi$ to be the subspace $\mathcal{D}_{\pi}=\overline{\operatorname{span}}\left\{\Theta_{\xi}(H): \xi \in \mathcal{B}_{\pi}\right\}$ of $\ell^{2}(G)$. Let $N$ be a positive integer. We call $N$ the orthogonality index of $\pi$ if $N$ is the smallest natural number such there exist $N$ strongly disjoint vectors $\xi_{i} \in \mathcal{B}_{\pi}$ such $\left\{\Theta_{\xi_{i}}\left(\mathcal{B}_{\pi}\right): i=1, \ldots, N\right\}$ 
generates $\mathcal{D}_{\pi}$. We say that $\pi$ has the orthogonality index $\infty$ if such a finite integer $N$ does not exist.

While in general it is very hard to find the orthogonality index for a given projective unitary representation, we are able, with the help of our first main result (Theorem 1.2), to prove the following theorem which, we believe, should be helpful in some cases.

Theorem 1.3. Let $\pi$ be a projective representation of a countable group $G$ on a Hilbert space $H$ such that $\mathcal{B}_{\pi}$ is dense in $H$. Then $\pi$ has the orthogonality index $N$ if and only if $\pi(G)^{\prime}$ has the cyclic multiplicity $N$, where $\pi(G)^{\prime}$ denotes the commutant of $\pi(G)$

The rest of the paper will be organized as follows: Section 2 will be devoted to proving our two theorems. We then discuss a few applications of these results in section 3. In particular, we give a duality result for a pair of projective unitary representations (Proposition 3.3). A special case is the known result in Gabor analysis (cf. $[5,6,17,25])$ which states that a Gabor sequence is a complete sequence in $L^{2}\left(\mathbb{R}^{d}\right)$ if and only if the corresponding adjoint Gabor sequence is $\ell^{2}$-linearly independent. In addition to the Gabor representation example, we also discuss some other examples demonstrating the applications of the main results.

\section{Proofs of the Main Results}

In order to prove Theorem 1.2 we needs several lemmas.

Lemma 2.1. [12] Let $\pi$ be a projective representation of a countable group $G$ on a Hilbert space $H$ such that $\mathcal{B}_{\pi}$ is dense in $H$. Then for any $x \in H$, there exists $\xi \in \mathcal{B}_{\pi}$ such that

(i) $\{\pi(g) \xi: g \in G\}$ is a Parseval frame for $[\pi(G) x]$;

(ii) $\Theta_{\xi}(H)=\left[\Theta_{x}\left(\mathcal{B}_{\pi}\right)\right]$.

Proof. Since this is an essential result to this paper, we provide a sketch for the case when $x$ is a Bessel vector. Full details are contained in Theorem 2 in [12] and its proof, including the general case where the polar decomposition for unbounded linear operators ([24]) is used.

Let $\Theta_{x}$ be the analysis operator for $\{\pi(g) x\}_{g \in G}$, and $\Theta_{x}=V\left(\Theta_{x}^{*} \Theta_{x}\right)^{1 / 2}$ be its polar decomposition, where $V: H \rightarrow \ell^{2}(G)$ be a partial isometry with the initial space $M:=$ $[\pi(G) x]$ and the final (range) space $K:=\left[\Theta_{x}(H)\right]$. Then a direct calculation shows that $V \pi(g)=\lambda_{g} V$ holds for every $g \in G$, where $\lambda$ is the corresponding left regular projective unitary representation of $G$. So this operator $V$ induces an unitary equivalence between the two sub-representations $\left.\lambda\right|_{K}$ and $\left.\pi\right|_{M}$. Let $\xi=V^{*} \chi_{e}$. Then it can be verified that $\xi$ satisfies the requirements.

Lemma 2.2. (Theorem 2.10, [13]) Let $\pi$ be a projective representation of a countable group $G$ on a Hilbert space $H$ such that $\mathcal{B}_{\pi}$ is dense in $H$. Then

$$
\pi(G)^{\prime}=\overline{\operatorname{span}}^{W O T}\left\{\Theta_{\eta}^{*} \Theta_{\xi}: \xi, \eta \in \mathcal{B}_{\pi}\right\}
$$

where "WOT" denotes the closure in the weak operator topology.

Lemma 2.3. Let $\pi$ be a projective representation of a countable group $G$ on a Hilbert space H. Then

$$
\left\langle\Theta_{x}(\xi), \Theta_{y}(\eta)\right\rangle=\left\langle\Theta_{\eta}(y), \Theta_{\xi}(x)\right\rangle
$$

holds for any $\xi, \eta \in \mathcal{B}_{\pi}$ and $x, y \in H$. 
Proof. This follows from direct calculation:

$$
\begin{aligned}
\left\langle\Theta_{x}(\xi), \Theta_{y}(\eta)\right\rangle & =\sum_{g \in G}\langle\xi, \pi(g) x\rangle\langle\pi(g) y, \eta\rangle \\
& =\sum_{g \in G}\left\langle\pi(g)^{*} \xi, x\right\rangle\left\langle y, \pi(g)^{*} \eta\right\rangle \\
& =\sum_{g \in G} \overline{\mu\left(g, g^{-1}\right)} \mu\left(g, g^{-1}\right)\left\langle\pi\left(g^{-1}\right) \xi, x\right\rangle\left\langle y, \pi\left(g^{-1}\right) \eta\right\rangle \\
& =\sum_{g \in G}\left\langle y, \pi\left(g^{-1}\right) \eta\right\rangle\left\langle\pi\left(g^{-1}\right) \xi, x\right\rangle \\
& =\left\langle\Theta_{\eta}(y), \Theta_{\xi}(x)\right\rangle .
\end{aligned}
$$

We divide the proof of Theorem 1.2 into two separate propositions.

Proposition 2.4. Let $\pi$ be a projective representation of a countable group $G$ on a Hilbert space $H$ such that $\mathcal{B}_{\pi}$ is dense in $H$, and let $x, y \in H$. Then the following are equivalent:

(i) $\Theta_{x}\left(\mathcal{B}_{\pi}\right) \perp \Theta_{y}\left(\mathcal{B}_{\pi}\right)$;

(ii) $\left[\pi(G)^{\prime} x\right] \perp\left[\pi(G)^{\prime} y\right]$.

Proof. By Lemma 2.2, we have that $\pi(G)^{\prime}$ is the weak operator closure of the linear span of $\left\{\Theta_{\eta}^{*} \Theta_{\xi}: \eta, \xi \in \mathcal{B}_{\pi}\right\}$. Thus we have that $\left[\pi(G)^{\prime} x\right] \perp\left[\pi(G)^{\prime} y\right]$ if and only if $\left\langle y, \Theta_{\eta}^{*} \Theta_{\xi}(x)\right\rangle=0$ for all $\xi, \eta \in \mathcal{B}_{\pi}$. However, from Lemma 2.3 , we have

$$
\left\langle\Theta_{x}(\xi), \Theta_{y}(\eta)\right\rangle=\left\langle\Theta_{\eta}(y), \Theta_{\xi}(x)\right\rangle=\left\langle y, \Theta_{\eta}^{*} \Theta_{\xi}(x)\right\rangle
$$

for all $\xi, \eta \in \mathcal{B}_{\pi}$. Thus, we obtain that $\left[\pi(G)^{\prime} x\right] \perp\left[\pi(G)^{\prime} y\right]$ if and only if $\Theta_{x}\left(\mathcal{B}_{\pi}\right) \perp \Theta_{y}\left(\mathcal{B}_{\pi}\right)$, and hence $(i) \Leftrightarrow(i i)$.

Proposition 2.5. Let $\pi$ be a projective representation of a countable group $G$ on a Hilbert space $H$ such that $\mathcal{B}_{\pi}$ is dense in $H$, and let $x, y \in H$. Then the following are equivalent:

(i) $\left[\Theta_{x}\left(\mathcal{B}_{\pi}\right)\right]=\left[\Theta_{y}\left(\mathcal{B}_{\pi}\right)\right]$

(ii) $\left[\pi(G)^{\prime} x\right]=\left[\pi(G)^{\prime} y\right]$.

Proof. $(i) \Rightarrow(i i)$ : Assume that $\left[\Theta_{x}\left(\mathcal{B}_{\pi}\right)\right]=\left[\Theta_{y}\left(\mathcal{B}_{\pi}\right)\right]$. Let $z \perp\left[\pi(G)^{\prime} x\right]$. Then, from Proposition 2.4, we have $\left[\Theta_{z}\left(\mathcal{B}_{\pi}\right)\right] \perp\left[\Theta_{x}\left(\mathcal{B}_{\pi}\right)\right]$. Hence $\left[\Theta_{z}\left(\mathcal{B}_{\pi}\right)\right] \perp\left[\Theta_{y}\left(\mathcal{B}_{\pi}\right)\right]$. Using Proposition 2.4 again we get $z \perp\left[\pi(G)^{\prime} y\right]$. Therefore we obtain that $\left[\pi(G)^{\prime} y\right] \subseteq\left[\pi(G)^{\prime} x\right]$. By symmetry we get the equality.

$(i i) \Rightarrow(i)$ : Assume that $\left[\pi(G)^{\prime} x\right]=\left[\pi(G)^{\prime} y\right]$. Let $P_{x}$ (resp. $P_{y}$ ) be the orthogonal projection from $\ell^{2}(G)$ onto $\left[\Theta_{x}\left(\mathcal{B}_{\pi}\right)\right]$ (resp. $\left[\Theta_{y}\left(\mathcal{B}_{\pi}\right)\right]$ ), and let $\lambda$ be the left regular $\mu$ projection representation of $G$, where $\mu$ is the multiplier of $\pi$. It is a routine exercise to check that both $\left[\Theta_{x}\left(\mathcal{B}_{\pi}\right)\right]$ and $\left[\Theta_{y}\left(\mathcal{B}_{\pi}\right)\right]$ are invariant under $\lambda$. Thus we have $P_{x} \lambda_{g}=\lambda_{g} P_{x}$ and $P_{y} \lambda_{g}=\lambda_{g} P_{y}$ for all $g \in G$.

We first show that $\left[\Theta_{y}\left(\mathcal{B}_{\pi}\right)\right] \subseteq\left[\Theta_{x}\left(\mathcal{B}_{\pi}\right)\right]$. Assume, to the contrary, that $P_{y} P_{x}^{\perp} \neq 0$. Then

$$
P_{y} P_{x}^{\perp} \chi_{e} \neq 0
$$


(since otherwise we would have $P_{y} P_{x}^{\perp} \chi_{g}=\lambda_{g} P_{y} P_{x}^{\perp} \chi_{e}=0$ and so $P_{y} P_{x}^{\perp}=0$ ), where $e$ is the group unit of $G$.

By Lemma 2.1, there exists $\eta \in \mathcal{B}_{\pi}$ such that $\{\pi(g) \eta: g \in G\}$ is a Parseval frame for $[\pi(G) y]$, and $\Theta_{\eta}(H)=\left[\Theta_{y}\left(\mathcal{B}_{\pi}\right)\right]$. So we have $\Theta_{\eta} \Theta_{\eta}^{*}=P_{y}$. Let $z=\Theta_{\eta}^{*} P_{y} P_{x}^{\perp} \chi_{e}$. Then, for any $w \in H$, we have

$$
\begin{aligned}
\Theta_{z}(w) & =\sum_{g \in G}\left\langle w, \pi(g) \Theta_{\eta}^{*} P_{y} P_{x}^{\perp} \chi_{e}\right\rangle \chi_{g} \\
& =\sum_{g \in G}\left\langle w, \Theta_{\eta}^{*} \lambda_{g} P_{y} P_{x}^{\perp} \chi_{e}\right\rangle \chi_{g} \\
& =\sum_{g \in G}\left\langle\Theta_{\eta}(w), P_{y} P_{x}^{\perp} \lambda_{g} \chi_{e}\right\rangle \chi_{g} \\
& =\sum_{g \in G}\left\langle P_{x}^{\perp} P_{y} \Theta_{\eta}(w), \chi_{g}\right\rangle \chi_{g} \\
& =P_{x}^{\perp} P_{y} \Theta_{\eta}(w)=P_{x}^{\perp} \Theta_{\eta}(w) .
\end{aligned}
$$

Therefore we get $\Theta_{z}(H) \perp\left[\Theta_{x}\left(\mathcal{B}_{\pi}\right)\right]$. Hence, by Proposition 2.4, we have $z \perp\left[\pi(G)^{\prime} x\right]$.

Since $P_{y} P_{x}^{\perp} \neq 0$, we have that $P_{x}^{\perp} P_{y} \neq 0$. So there exist $w \in H$ such that $P_{x}^{\perp} \Theta_{\eta}(w)=$ $P_{x}^{\perp} P_{y} \Theta_{\eta}(w) \neq 0$, which implies that

$$
0 \neq\left\langle P_{x}^{\perp} \Theta_{\eta}(w), P_{x}^{\perp} \Theta_{\eta}(w)\right\rangle=\left\langle P_{x}^{\perp} \Theta_{\eta}(w), \Theta_{\eta}(w)\right\rangle=\left\langle\Theta_{z}(w), \Theta_{\eta}(w)\right\rangle .
$$

Hence

$$
\Theta_{z}(w) \notin\left[\Theta_{\eta}(H)\right]^{\perp}=\left[\Theta_{y}\left(\mathcal{B}_{\pi}\right)\right]^{\perp} .
$$

Therefore, by Proposition 2.4, $z$ is not orthogonal to $\left[\pi(G)^{\prime} y\right]$. But we already have that $z \perp\left[\pi(G)^{\prime} x\right]$ and $\left[\pi(G)^{\prime} x\right]=\left[\pi(G)^{\prime} y\right]$. This is a contradiction. Therefore we must have $\left[\Theta_{y}\left(\mathcal{B}_{\pi}\right)\right] \subseteq\left[\Theta_{x}\left(\mathcal{B}_{\pi}\right)\right]$. By symmetry we also have $\left[\Theta_{x}\left(\mathcal{B}_{\pi}\right)\right] \subseteq\left[\Theta_{y}\left(\mathcal{B}_{\pi}\right)\right]$. Therefore we get (i).

Clearly, the proof of the above proposition also implies the following more general statement:

Proposition 2.6. Let $\pi$ be a projective representation of a countable group $G$ on a Hilbert space $H$ such that $\mathcal{B}_{\pi}$ is dense in $H$, and let $x, y \in H$. Then the following are equivalent:

(i) $\left[\Theta_{y}\left(\mathcal{B}_{\pi}\right)\right] \subseteq\left[\Theta_{x}\left(\mathcal{B}_{\pi}\right)\right]$

(ii) $\left[\pi(G)^{\prime} y\right] \subseteq\left[\pi(G)^{\prime} x\right]$.

Proof of Theorem 1.2: This immediately follows from Proposition 2.4 and Proposition 2.5 .

In order to prove the second theorem we need the following result:

Proposition 2.7. Let $\pi$ be a projective representation of a countable group $G$ on a Hilbert space $H$ such that $\mathcal{B}_{\pi}$ is dense in $H$. Then the following are equivalent;

(i) $\pi$ has the orthogonality index $N$;

(ii) $N$ is the smallest number such that there exist $N$-strongly disjoint Parseval frame vectors $\left\{\xi_{i}: 1 \leq i \leq N\right\}$ such that $D_{\pi}=\sum_{i=1}^{N} \oplus \Theta_{\xi_{i}}(H)$. 
Proof. From the definition of the orthogonality index, clearly it is sufficient to show that if $\left\{x_{i}: 1 \leq i \leq N\right\}$ are $\pi$-orthogonal vectors such that $D_{\pi}=\sum_{i=1}^{N} \oplus\left[\Theta_{x_{i}}(H)\right]$, then there exist $\pi$-orthogonal Parseval frame vectors $\left\{\xi_{i}: 1 \leq i \leq N\right\}$ such that $D_{\pi}=\sum_{i=1}^{N} \oplus \Theta_{\xi_{i}}(H)$. However, this follows from Lemma 2.1 since for each $i$ there exists $\xi_{i}$ such that $\left\{\pi(g) \xi_{i}\right\}_{g \in G}$ is a Parseval frame for $\left[\pi(G) x_{i}\right]$ and $\Theta_{\xi_{i}}(H)=\left[\Theta_{x_{i}}(H)\right]$.

\section{Proof of Theorem 1.3:}

Let $N$ be the orthogonality index for $\pi$ and $K$ be the cyclic multiplicity of $\pi(G)^{\prime}$.

We first prove that $K \leq N$. By Proposition 2.7, there exist $\pi$-orthogonal Parseval frame vectors $\left\{\xi_{i}: 1 \leq i \leq N\right\}$ such that $\mathcal{D}_{\pi}=\sum_{i=1}^{N} \oplus \Theta_{\xi_{i}}(H)$. We claim that $\left\{\xi_{i}: 1 \leq i \leq N\right\}$ is a cyclic set for $\pi(G)^{\prime}$. In fact, assume to the contrary that $\overline{\operatorname{span}}\left\{\pi(G)^{\prime} \xi_{i}: g \in G, 1 \leq i \leq\right.$ $N\} \neq H$. Then there exists $x \neq 0$ such that $\left[\pi(G)^{\prime} x\right] \perp \overline{\operatorname{span}}\left\{\pi(G)^{\prime} \xi_{i}: g \in G, 1 \leq i \leq N\right\}$. Hence, by Proposition 2.4, $\Theta_{x}\left(\mathcal{B}_{\pi}\right) \perp \Theta_{\xi_{i}}(H)$ for all $1 \leq i \leq N$, which contradicts to the assumption that $\mathcal{D}_{\pi}=\sum_{i=1}^{N} \oplus \Theta_{\xi_{i}}(H)$. Thus $\left\{\xi_{i}: 1 \leq i \leq N\right\}$ is a cyclic set for $\pi(G)^{\prime}$ and so $K \leq N$.

Next we show that $N \leq K$. Since $\pi(G)^{\prime}$ has the cyclic multiplicity $K$, there exist $K$ vectors $x_{i} \in H$ such $\overline{\operatorname{span}}\left\{\pi(G)^{\prime} x_{i}: 1 \leq i \leq K\right\}=H$ and $\left[\pi(G)^{\prime} x_{i}\right] \perp\left[\pi(G)^{\prime} x_{j}\right]$ when $i \neq j$. By Theorem 1.2 we know that $\left\{x_{i}\right\}_{i=1}^{k}$ are mutually $\pi$-orthogonal vectors. To show that $N \leq K$, it suffices to prove that $\left\{\Theta_{x_{i}}\left(\mathcal{B}_{\pi}\right): 1 \leq i \leq K\right\}$ generates $\mathcal{D}_{\pi}$. Let $M:=\overline{\operatorname{span}}\left\{\Theta_{x_{i}}\left(\mathcal{B}_{\pi}\right): 1 \leq i \leq K\right\}$, and let $x \in H$ be arbitrary. We need to show that $\left[\Theta_{x}\left(\mathcal{B}_{\pi}\right)\right] \subseteq M$. In fact, let $P_{i}$ be the orthogonal projection from $H$ onto $\left[\pi(G)^{\prime} x_{i}\right]$. Then $P_{i}$ commutes with $\pi(G)^{\prime}$ and $x=\sum_{i=1}^{K} P_{i} x$.

Since $P_{i} x \in\left[\pi(G)^{\prime} x_{i}\right]$, we have that $\left[\pi(G)^{\prime} P_{i} x\right] \subseteq\left[\pi(G)^{\prime} x_{i}\right]$. Thus , by Proposition 2.6, we obtain $\left[\Theta_{P_{i} x}\left(\mathcal{B}_{\pi}\right)\right] \subseteq\left[\Theta_{x_{i}}\left(\mathcal{B}_{\pi}\right)\right]$. This implies that for each $\xi \in \mathcal{B}_{\pi}$,

$$
\Theta_{x}(\xi)=\sum_{i=1}^{K} \Theta_{P_{i} x}(\xi) \in M
$$

and so $\left[\Theta_{x}\left(\mathcal{B}_{\pi}\right)\right] \subseteq M$. Therefore we get $M=\mathcal{D}_{\pi}$, as claimed. Thus $N \leq K$.

\section{Some Applications}

In this section we briefly discuss a few applications of the main results. We first examine our motivating example: Gabor representations. For $(t, s) \in \mathbb{R}^{d} \times \mathbb{R}^{d}$, define the timefrequency representation (Gabor representation) $\pi$ by:

$$
\pi(t, s)=E_{s} T_{t}
$$

where $E_{s} f(x)=e^{2 \pi i<x, s>}$ and $T_{t} f(x)=f(x-t)$ for $f \in L^{2}\left(\mathbb{R}^{d}\right)$. Let $G$ be a full rank lattice in $\mathbb{R}^{d} \times \mathbb{R}^{d}$ (i.e., $G=M\left(\mathbb{Z}^{d} \times \mathbb{Z}^{d}\right)$ for some invertible real matrix M). The adjoint lattice of $G$ is defined to be the lattice $\tilde{G}=\left\{x \in \mathbb{R}^{d} \times \mathbb{R}^{d}:\langle\lambda, x\rangle \in \mathbb{Z}, \forall \lambda \in G\right\}$. In the case that a lattice $G=A \mathbb{Z}^{d} \times B \mathbb{Z}^{d}$ is separable, the adjoint lattice of $G$ is given by $\tilde{G}=\tilde{\mathcal{K}} \times \tilde{\mathcal{L}}=\left(B^{t}\right)^{-1} \mathbb{Z}^{d} \times\left(A^{t}\right)^{-1} \mathbb{Z}^{d}$. The volume of a full rank lattice $G=M\left(\mathbb{Z}^{d} \times \mathbb{Z}^{d}\right)$ is given by $\mathrm{v}(G)=|\operatorname{det} M|$.

For a full rank lattice $G$ in $\mathbb{R}^{d} \times \mathbb{R}^{d}$, the Gabor Representation $\left.\pi\right|_{G}$ is a projective unitary representation of $G$ with the property that the Bessel vectors for $\left.\pi\right|_{G}$ is dense in $L^{2}\left(\mathbb{R}^{d}\right)$, 
and $\mathcal{D}_{\left.\pi\right|_{G}}=\ell^{2}(G)$. It is known that (cf. [6]) that $\pi(G)^{\prime}$ is exactly the von Neumann algebra generated by $\pi(\tilde{G})$. Moreover, it is trivial to check that in the one dimensional $(d=1)$ case $\pi(G)$ has the cyclic multiplicity $N$ if and only if $N-1<|\mathrm{v}(G)| \leq N$. This is also true for the high dimension and separable lattice cases (it follows from the proofs of lattice tiling and packing results obtained in $[21,22]$ and [19]). Therefore, from Theorem 1.3, we immediately have the following consequence:

Corollary 3.1. Let $G=A \mathbb{Z}^{d} \times B \mathbb{Z}^{d}$ be a full rank lattice in $\mathbb{R}^{d} \times \mathbb{R}^{d}$. Then the Gabor representation $\left.\pi\right|_{G}$ has the orthogonality index $N$ if and only if $\frac{1}{N} \leq \mathrm{v}(G)<\frac{1}{N-1}$.

We believe that the above corollary also holds for any non-separable lattices. However, our techniques used in [21] and [19] only apply to the case when $G$ is a separable lattice. It is possible that the techniques used in [4] may lead to a proof for the non-separable lattice cases. We remark that in the separable $G=A \mathbb{Z}^{d} \times B \mathbb{Z}^{d}$ case, if $\frac{1}{N}<|\operatorname{det}(A B)|<\frac{1}{N-1}$, then the frame multiplicity of $\left.\pi\right|_{G}$ is $N-1$, while by Corollary 3.1, the orthogonality index is $N$. The two agree if and only if $|\operatorname{det}(A B)|=\frac{1}{N}$ for some positive integer $N$.

One of the well-known properties for Gabor frames is the so-called duality principle (most commonly referenced as the Ron-Shen Duality theorem, cf. [6, 23, 25]) involving different class of vectors (e.g., frame vectors, Bessel vectors, cyclic vectors, Riesz sequence generators). For example, the duality principle states that (i) $\psi$ is a complete frame vector for $\left.\pi\right|_{G}$ if and only if $\{\pi(\tilde{g}) \psi: \tilde{g} \in \tilde{G}\}$ is a Riesz sequence, and (ii) if $\psi$ is Bessel, then the Gabor sequence $\{\pi(g) \psi: g \in G\}$ is complete in $L^{2}\left(\mathbb{R}^{d}\right)$ if and only if the adjoint of the analysis operator $\{\pi(\tilde{g}) \psi: \tilde{g} \in \tilde{G}\}$ is injective. We point out that Theorem 1.2 provides an alternate proof for $(i i)$. In fact, it generalizes to non-Bessel vector cases.

Corollary 3.2. Let $G$ be a full rank lattice in $\mathbb{R}^{d} \times \mathbb{R}^{d}$. Then $\{\pi(g) \psi: g \in G\}$ is complete sequence in $L^{2}\left(\mathbb{R}^{d}\right)$ if and only if $\{\pi(\tilde{g}) \psi: \tilde{g} \in \tilde{G}\}$ is $\ell^{2}$-linearly independent, where by the $\ell^{2}$-linearly independence of $\{\pi(\tilde{g}) \psi: \tilde{g} \in \tilde{G}\}$ we mean that whenever $c:=\left(c_{\tilde{g}}\right) \in \ell^{2}(\tilde{G})$ satisfies the condition that $\sum_{\tilde{g} \in \tilde{G}} c_{\tilde{g}}\langle\pi(\tilde{g}) \psi, \eta\rangle$ is convergent to 0 for all $\eta \in \mathcal{B}_{\pi_{\tilde{G}}}$, then we have that $c=0$.

This is actually a special case of the following more general result:

Proposition 3.3. Let $\pi$ and $\tau$ be two projective unitary representations of a group $G$ on the same Hilbert space $H$. Assume that $\mathcal{B}_{\tau}$ dense in $H, \tau(G)$ generates the von Neumann algebra $\pi(G)^{\prime}$ and $\mathcal{D}_{\tau}=\ell^{2}(G)$. Then $\{\pi(g) \psi: g \in G\}$ is a complete sequence in $H$ if and only if $\{\tau(g) \psi: g \in G\}$ is $\ell^{2}$-linearly independent.

Proof. First assume that $\{\pi(g) \psi: g \in G\}$ is a complete sequence in $H$. Then, by Theorem 1.2 , we have that there is no non-zero vector $y \in H$ such that $\psi$ and $y$ are $\tau$-orthogonal. This implies that $\left[\Theta_{\psi}\left(\mathcal{B}_{\tau}\right)\right]=\mathcal{D}_{\tau}=\ell^{2}(G)$. If $c=\left\{c_{g}\right\}_{g \in G} \in \ell^{2}(G)$ such that $\sum_{g \in G} c_{g} \tau(g) \psi=0$, then we have that $\left\langle c, \Theta_{\psi}(\eta)\right\rangle=0$ for all $\eta \in \mathcal{B}_{\tau}$. Hence $c=0$. Hence $\{\tau(g) \psi: g \in G\}$ is $\ell^{2}$-linearly independent, as claimed.

Conversely, assume that $\{\tau(g) \psi: g \in G\}$ is $\ell^{2}$-linearly independent. We need to show that $\left[\tau(G)^{\prime} \psi\right]=H$. Assume, otherwise, there is $0 \neq \eta \perp\left[\tau(G)^{\prime} \psi\right]$ and $\eta \in H$. Then, by Theorem 1.2 again, we get $\left[\Theta_{\psi}\left(\mathcal{B}_{\tau}\right)\right] \perp\left[\Theta_{\eta}\left(\mathcal{B}_{\tau}\right)\right]$. Thus $\left[\Theta_{\psi}\left(\mathcal{B}_{\tau}\right)\right] \neq \ell^{2}(G)$, which implies that $\{\tau(g) \psi: g \in G\}$ is not $\ell^{2}$-linearly independent. So we have $H=\left[\tau(G)^{\prime} \psi\right]=[\pi(G) H]$ and therefore $\{\pi(g) \psi: g \in G\}$ is a complete sequence in $H$ 
The next two examples give additional applications.

Example 3.1. Let $\pi$ be a projective unitary representation such that $\mathcal{B}_{\pi}$ is dense in $H$. If there exists $\xi \in H$ such that $\{\pi(g) \xi: g \in G\}$ is a Riesz sequence, then $\pi(G)^{\prime}$ has the cyclic multiplicity one. In fact, in this case we have $\Theta_{\xi}(H)=\mathcal{D}_{\pi}$ and hence $\pi$ has the orthogonality index one. Moreover, $\left[\pi(G)^{\prime} \xi\right]=H$.

Example 3.2. Let $\pi$ be a $\mu$-projective unitary representation of $G$ on $H$ which admits a complete frame vector $\xi$.

(i) If $\pi(G)$ is abelian, then $\mathcal{D}_{\pi}=\Theta_{\xi}(H)$ and the orthogonality index of $\pi$ is 1 .

(ii) If $G$ is an ICC-group (or more generally, the von Neumann algebra $\mathcal{L}$ generated by the left regular $\mu$-projective unitary representation is a factor), then $\mathcal{D}_{\pi}=\ell^{2}(G)$ and the orthogonality index of $\pi$ is $N$ if $\frac{1}{N} \leq\|\xi\|^{2}<\frac{1}{N-1}$. Moreover, the orthogonality index and the frame multiplicity agree only when $\|\xi\|^{2}=\frac{1}{N}$ for some integer $N$.

Part (i) is obvious from Theorem 1.3. While part (ii) is a consequence of Theorem 1.3 and the fact that two projections in a factor von Neumann algebra are equivalent if and only if they have same trace (cf. [24]).

The following characterizes all the projective unitary representations which admit complete frame vectors and have orthogonality index one.

Proposition 3.4. Let $\pi$ be a $\mu$-projective unitary representation of $G$ on $H$ which admits a complete frame vector $\xi$. Then following are equivalent:

(i) $\pi$ has orthogonality index one;

(ii) The orthogonal projection $P_{\xi}$ onto $\Theta_{\xi}(H)$ is in the center of $\mathcal{L}$, where $\mathcal{L}$ is the von Neumann algebra generated by $\lambda$ with multiplier $\mu$;

(iii) For every complete frame vector $\eta$ for $\pi$, there exists an invertible operator $T \in \pi(G)^{\prime}$ such that $\eta=T \xi$;

(iv) $\xi$ is a cyclic vector for $\pi(G)^{\prime}$;

(v) For every complete frame vector $\eta$ for $\pi$, there exits a unique frame vector $\psi$ for $\pi$ such that

$$
x=\sum_{g \in G}\langle x, \pi(g) \xi\rangle \pi(g) \psi
$$

holds for every vector $x \in H$.

Proof. The equivalence from $(i i)$ to $(v)$ was proved in [15] (Theorem 3.6). From Theorem 1.3 , we have that $(i v)$ implies $(i)$. So it suffices to show that $(i)$ implies $(v)$. Assume that $\pi$ has the orthogonality index one. Then there exists $x \in H$ such that $\left[\Theta_{x}\left(\mathcal{B}_{\pi}\right)\right]=\mathcal{D}_{\pi}$. Using Proposition 2.1, there exists $\eta$ such that $\left[\Theta_{x}\left(\mathcal{B}_{\pi}\right]=\Theta_{\eta}(H)\right.$ and $\{\pi(g) \eta\}_{g \in G}$ is a Parserval frame for $[\pi(G) x](=[\pi(G) \eta])$.

We first show that $[\pi(G) x]=H$. In fact, similar to the proof of Theorem 4 in [12], it can be shown that, there exists vector $\varphi$ such that $\eta+\varphi$ is a complete frame vector for $\pi$ and $\eta, \varphi$ are $\pi$-orthogonal. So we have $\Theta_{\varphi}(H) \perp \Theta_{\eta}(H)=\mathcal{D}_{\pi}$, and hence $\varphi=0$. Therefore $[\pi(G) x]=[\pi(G) \eta]=H$.

Secondly, we prove that $\left[\pi(G)^{\prime} \eta\right]=H$. Assume, otherwise, $\left[\pi(G)^{\prime} \eta\right] \neq H$. Then there exists $0 \neq y$ such that $\left[\pi(G)^{\prime} \eta\right] \perp\left[\pi(G)^{\prime} y\right]$ and so, by Theorem 1.2 , we have $\mathcal{D}_{\pi}=\left[\Theta_{\eta}\left(\mathcal{B}_{\pi}\right)\right] \perp$ 
$\left[\Theta_{y}\left(\mathcal{B}_{\pi}\right)\right] \subseteq \mathcal{D}_{\pi}$, which is a contradiction. Therefore $\left[\pi(G)^{\prime} \eta\right]=H$ and hence $\eta$ is a complete Parserval frame vector for $\pi$.

By the parameterization theorem (cf. $[18,20]$ ), there exists an invertible operator $T$ in the von Neumann algebra generated by $\pi(G)$ such that $\xi=T \eta$. Thus we get $(i v)$ since $[\pi(G) \xi]=[\pi(G) T \eta]=[\pi(G) \eta]=H$.

Any frame vector $\varphi$ satisfying the reconstruction formula in $(v)$ of Proposition 3.4 is called a dual frame vector for $\xi$. The above proposition tells us that orthogonality index characterizes all the frame vectors that have a unique dual frame vector (we remark that since these frames are not necessarily Riesz bases, usually they still have duals of other kind. But the dual frame vector is unique when $\pi$ has the orthogonality index one). On the other side if the orthogonality index is higher enough then we may have a better choices for the dual frame vectors. For example we have the following:

Proposition 3.5. Let $\pi$ be a $\mu$-projective unitary representation of $G$ on $H$ which admits a complete frame vector. Assume that the von Neumann algebra generated by the left regular $\mu$-projective unitary representation is a factor and the orthogonality index is at least 3 . Then for any complete frame vector $\xi$ of $\pi$, there exists a complete tight frame vector $\eta$ such that $\eta$ is a dual frame vector of $\xi$.

Proof. The assumption implies that there exists two $\pi$-orthogonal frame vectors for $\pi$, and so the conclusion follows from Theorem 1.2 in [19].

Acknowledgement. The authors thank the referee for several valuable comments and suggestions.

\section{REFERENCES}

[1] A. Aldroubi, D. Larson, Wai-Shing Tang and E. Weber, Geometric aspects of frame representations of abelian groups, Trans. Amer. Math. Soc., 356 (2004), no. 12, 4767-4786

[2] R. Balan, Equivalence relations and distances between Hilbert frames, Proc. Amer. Math. Soc., 127 (1999), 2353-2366.

[3] R. Balan, A study of Weyl-Heisenberg and wavelet frames, Ph. D. Thesis, Princeton University, 1998.

[4] B. Bekka Square integrable representations, von Neumann algebras and an application to Gabor analysis, J. Fourier Anal. Appl., 10(2004), 325-349.

[5] P. Casazza, G. Kutyniok and M. Lammers, Duality principles in frame theory, J. Fourier Anal. Appl., 10 (2004), 383-408.

[6] I. Daubechies, H. Landau and Z. Landau, Gabor time-frequency lattices and the Wexler-Raz identity, J. Fourier Anal. Appl., 1 (1995), 437-478.

[7] R. J. Duffin and A. C. Schaeffer, A class of nonharmonic Fourier series, Trans. Amer. Math. Soc., 72(1952), 341-366.

[8] D. Dutkay, Ervin Positive definite maps, representations and frames, Rev. Math. Phys., 16 (2004), no. 4, 451-477.

[9] D. Dutkay, The local trace functions for super-wavelets Contemp. Math., 345 (2004), 115-136.

[10] D. Dutkay, S. Bildea and G. Picioroaga, MRA Superwavelets, New York Journal of Mathematics , 11 (2005), 1-19.

[11] D. Dutkay and P. Jorgensen, Oversampling generates super-wavelets, Proc. Amer. Math. Soc., 135 (2007), no. 7, 2219-2227. 
[12] J-P. Gabardo and D. Han, Subspace Weyl-Heisenberg frames, J. Fourier Anal. Appl., 7 (2001), 419-433.

[13] J-P. Gabardo and D. Han, Frame representations for group-like unitary operator systems, J. $O p$ erator Theory, 49(2003), 223-244.

[14] J-P. Gabardo and D. Han, Aspects of Gabor analysis and operator algebras. Advances in Gabor analysis, 129-152, Appl. Numer. Harmon. Anal., Birkhuser Boston, Boston, MA, 2003

[15] J-P. Gabardo and D. Han, The uniqueness of the dual of Weyl-Heisenberg subspace frames, Appl. Comput. Harmon. Anal., 17 (2004), 226-240.

[16] J-P. Gabardo and D. Han, Balian-Low phenomenon for subspace Gabor frames, J. Math. Phys., 45 (2004), 3362-3378.

[17] K. Gröchenig, Foundations of Time-Frequency Analysis, Applied and Numerical Harmonic Analysis, Birkhäuser, 2001.

[18] D. Han, Approximations for Gabor and wavelet frames, Trans. Amer. Math. Soc., 355 (2003), 3329-3342.

[19] D. Han, Frame Representations and Parseval Duals with Applications to Gabor Frames, Trans. Amer. Math. Soc., 360(2008), 3307-3326.

[20] D. Han and D. Larson, Frames, bases and group representations, Memoirs Amer. Math. Soc., 697 (2000).

[21] D. Han and Y. Wang, Lattice tiling and Weyl-Heisenberg frames, Geometric and Functional Analysis, 11(2001), 742-758.

[22] D. Han and Y. Wang, The existence of Gabor bases, Contemp. Math., 345 (2004), 183-192.

[23] C. Heil, History and evolution of the density theorem for Gabor frames, J. Fourier Anal. Appl., 13 (2007), 113-166.

[24] R. Kadison and J. Ringrose, Fundamentals of the Theory of Operator Algebras, Vol. I and II, Academic Press, Inc. 1983 and 1985.

[25] A. Ron and Z. Shen, Weyl-Heisenberg frames and Riesz bases in $L_{2}\left(\mathbb{R}^{d}\right)$, Duke Math. J., 89(1997) $237-282$.

[26] V. S. Varadarajan, Geometry of Quantum Theory, Second Edition, Springer-Verlag, New YorkBerlin, 1985.

[27] E. Weber, Orthogonal frames of translates, Appl. Comput. Harmon. Anal., 17 (2004), 69-90.

Department of Mathematics, University of Central Florida, Orlando, FL 32816

E-mail address: dhan@pegasus.cc.ucf.edu

Department of Mathematics, Texas A\&M University, College Station, TX

E-mail address: larson@math.tamu.edu 